\title{
Atuação do médico generalista frente à violência sexual contra crianças e adolescentes
}

\author{
Generalist doctor action against sexual violence against children and teenagers
}

Actuación del médico generalista frente a la violencia sexual contra niños y adolescentes

Maria Augusta Cunha Menezes ${ }^{1}$, Rita de Cássia Natividade Ataíde ${ }^{1}$, Ana Carolina da Silva Soares Martins $^{1}$, Ana Maria Romani ${ }^{2}$, Carla Gabriela Rodrigues Cavalcante ${ }^{3}$, Lara Coimbra de Oliveira ${ }^{3}$, Nayara Máximo Carvalho Silva ${ }^{3}$, Tárcia Gomes Silva ${ }^{3}$, Karlla Susane Costa Monteiro ${ }^{4}$, Valmir Martins Falcão Neto 5 .

\section{RESUMO}

Objetivo: Compreender como o médico generalista deve proceder diante dos casos de violência sexual contra crianças e adolescentes. Revisão bibliográfica: A violência sexual contra crianças e adolescentes é uma constante no mundo desde os tempos mais remotos, presentes em todas as culturas, raças e diferentes níveis socioeconômicos. Apesar do grave problema de saúde que esta violência representa, ainda é pouco discutida dentro da sociedade, considerando a magnitude dos traumas e sequelas impostos às suas vítimas. No Brasil, o dia 18 de maio é destinado ao combate à violência sexual contra crianças e adolescentes, entretanto, os registros desse tipo de crime contra menores de idade são crescentes, em que pese a subnotificação séria no país. Considerações finais: Ainda que existam políticas públicas que integrem as áreas da saúde e educação para fortalecimento do combate à violência sexual contra menores de idade, o diagnóstico é tardio. Além do patriarcalismo social que acaba interferindo nas denúncias contra os agressores por medo das consequências legais sobre os autores dos crimes, há também a falta de conhecimento dos profissionais da saúde sobre o tema no atendimento às vítimas.

Palavras-chave: Abuso sexual infantil, Violência sexual contra crianças e adolescentes, Medicina da família.

\begin{abstract}
Objective: Understand how the general doctor should deal with cases of sexual violence against children and teenagers. Bibliographic review: Sexual violence against children and teenagers is a constant in the world since ancient times, present in all cultures, races, and different socioeconomic levels. Despite the serious health problem that this violence represents, it is still little discussed within society, considering the magnitude of the trauma and sequelae imposed on its victims. In Brazil, May 18th is dedicated to fighting sexual violence against children and teenagers, however, the records of this type of crime against minors are growing, despite the serious underreporting in the country. Final considerations: Although there are public policies that integrate the areas of health and education to strengthen the fight against sexual violence against minors, the diagnosis is late. Besides the social patriarchy that interferes with the accusations against the aggressors for fear of the legal consequences on the perpetrators, there is also the lack of knowledge of health professionals on the subject when assisting the victims.
\end{abstract}

Key words: Child sexual abuse, Sexual violence against children and teenagers, Family medicine.

\footnotetext{
${ }^{1}$ Faculdade de Saúde Santo Agostinho de Vitória da Conquista (FASA - VIC), Vitória da Conquista - BA.

*E-mail: maugustacunha@outlook.com

2 Faculdades Integrada Aparício Carvalho (FIMCA), Porto Velho - RO.

${ }^{3}$ Centro Universitário de São Francisco de Barreiras (UNIFASB), Barreiras - BA.

${ }^{4}$ Centro Universitário Facid Wyden (Unifacid), Teresina - PI.

${ }^{5}$ União Educacional do Vale do Aço (UNIVAÇO), Ipatinga - MG.
} 


\section{RESUMEN}

Objetivo: Comprender cómo debe tratar el médico de cabecera los casos de violencia sexual contra niños y adolescentes. Reseña bibliográfica: La violencia sexual contra niños y adolescentes es una constante en el mundo desde los tiempos más remotos, presente en todas las culturas, razas y diferentes niveles socioeconómicos. A pesar del grave problema de salud que representa esta violencia, todavía se discute poco en la sociedad, teniendo en cuenta la magnitud del trauma y las secuelas que impone a sus víctimas. En Brasil, el 18 de mayo está dedicado a combatir la violencia sexual contra niños y adolescentes, sin embargo, los registros de este tipo de delitos contra menores están creciendo, a pesar del grave subregistro en el país. Consideraciones finales: Si bien existen políticas públicas que integran las áreas de salud y educación para fortalecer el combate a la violencia sexual contra menores, el diagnóstico es tardío. Al patriarcado social que interfiere en las denuncias contra los agresores por miedo a las consecuencias legales sobre los mismos, se suma el desconocimiento de los profesionales sanitarios sobre el tema en la atención a las víctimas.

Palabras clave: Abuso sexual infantil, Violencia sexual contra niños y adolescentes, Medicina familiar.

\section{INTRODUÇÃO}

Carregada de sofrimento e capaz de causar uma série de danos tanto físicos quanto psicológicos às suas vítimas, a violência sexual contra crianças e adolescentes é um importante problema de saúde pública no Brasil. Presente em toda a sociedade, afeta meninos e meninas de todas as faixas etárias, tanto em âmbito intrafamiliar, como extrafamiliar (BRASIL, 2018).

Os casos de violência sexual contra crianças e adolescentes é crescente em todo o mundo. O Ministério da Saúde registrou 32 mil casos no ano de 2018, mas que representam apenas $10 \%$ dos casos devido à grave subnotificação. O sub registro decorre principalmente da dificuldade da família em denunciar os agressores, por medo ou vergonha, pois muitas vezes o abuso é praticado por pessoas do convívio diário das vítimas (pai, padrasto, avô, irmão, primo), como também a pequena idade das vítimas, que pode levá-las, inclusive, a nem perceber que estão sendo violentadas (BRASIL, 2018). Diante disso, as vítimas de tal violência possuem "feridas afetivas" que não são cicatrizadas, uma vez que o ato é praticado por alguém que elas confiam (FLORENTINO BRB, 2015).

O Sistema de Informação de Agravos de Notificação (SINAN/MS) registrou, nos anos de 2011 a 2017, 184.524 casos de violência sexual. Destes, 141.105 (76,5\%) foram praticados contra crianças $(31,5 \%)$ e adolescentes $(45,0 \%)$. Estes números, entretanto, retratam apenas $10 \%$ dos casos de abuso sexual infanto juvenil no país, revelando a gravidade da subnotificação. A inexistência nos serviços de saúde de um instrumento de registro específico para a violência sexual contra menores agrava ainda mais a situação (BRASIL, 2018).

Diante desse cenário chocante, as vítimas de abuso sexual durante a infância e adolescência podem desencadear sequelas que carregarão pelo resto das suas vidas, a exemplo do transtorno de estresse póstraumático (TEPT), uso/abuso de drogas, depressão, dificuldade em manter uma gestação e até tentativa de suicídio (BRASIL, 2018).Também pode haver impacto dessa violência sobre seus familiares, nos relacionamentos futuros e no meio social em que vivem essas crianças e adolescentes (RIBEIRO MA, et al., 2004).

Os órgãos de proteção da criança e do adolescente (Fundo das Nações Unidas para a Infância (UNICEF), Conselho Tutelar, Justiça da Infância e Juventude, Ministério Público, Polícia Civil) vêm atuando no combater à violência sexual intradomiciliar. Observa-se que nos últimos meses, em virtude da reclusão social desencadeada pela pandemia da COVID - 19, as crianças e adolescentes encontram-se mais vulneráveis por estarem em contato diário permanente com adultos. Além disso, neste mesmo período, a rede de apoio encontra-se fragilizada (COSTA F, 2020).

No Brasil, o dia 18 de maio foi instituído o Dia Nacional de Combate ao Abuso e à Exploração Sexual de Crianças e Adolescentes pela Lei Federal 9.970/00. Esta lei tem por função dar visibilidade ao tema com o 
intuito de informar e fortalecer os seus princípios tanto no ambiente domiciliar, escolar, comunitário, como na sociedade (BRASIL, 2000).

O sistema público dispõe da Atenção Primária à Saúde, porta de entrada e ordenadora do cuidado, exercendo uma verdadeira capilaridade que permite acompanhar cada família através das equipes e dos Agentes Comunitários de Saúde (ACS) (BRASIL, 2017). Desta forma, o médico generalista, em especial, o médico da estratégia de saúde da família, constitui-se uma peça fundamental de atuação no combate ao crime sexual contra menores.

\section{REVISÃO BIBLIOGRÁFICA}

De origem multifatorial, a violência está associada a atos de constrangimento e uso de superioridade física sobre o outro, sendo variável de acordo com a época e lugar. Trata-se de um fenômeno da ordem de quem a vive, logo sua definição torna-se difícil, pois suas manifestações provocam ou são provocadas pela carga emocional de quem a comete, de quem a sofre e de quem a presencia (HORA TD e PAIVA AR, 2017).

Grave problema de saúde pública, a violência está presente na sociedade desde os tempos primitivos, em que já ocorriam atos violentos, desde um simples insulto até espancamentos, homicídios, exploração sexual e estupros. No decorrer dos tempos, tais atitudes tornaram-se atos criminosos graças à criação e implementação de leis para que a sociedade pudesse viver de forma democrática. Diante disso, é importante levar em consideração a violência centrada na criança e no adolescente visto ser crescente ao nível global e na maioria das vezes, os casos são subnotificados (SANTOS CO, et al., 2015).

A violência contra a criança e adolescente, segundo Minayo MSC (2001) é definida como todo ação ou omissão causado por pais, parentes, outras pessoas e instituições, capazes de gerar dano físico, sexual e/ou psicológico à vítima. A classificação de maus tratos contra crianças e adolescentes são divididos em violência física, violência emocional/ psicológica, abuso sexual e negligencia.

A Constituição Federal de 1988 em seu artigo 227, páginas 178 - 180, impõe:

"É dever da família, da sociedade e do Estado assegurar à criança, ao adolescente e ao jovem, com absoluta prioridade, o direito à vida, à saúde, à alimentação, à educação, ao lazer, à profissionalização, à cultura, à dignidade, ao respeito, à liberdade e à convivência familiar e comunitária, além de colocá-los a salvo de toda forma de negligência, discriminação, exploração, violência, crueldade e opressão" (BRASIL, 1988).

Mesmo sendo assegurado por lei, na década de 1980 após diversos estudos na área da saúde voltado para temática violência - saúde, observou-se a importância de implementar na legislação um projeto de lei voltado para a proteção da criança e do adolescente visto que eram crescentes os casos contra a sua integridade física e emocional. Diante disso, em 13 de julho de 1990 a constituição brasileira promulgou a Lei 8.069/12, a chamada Lei do Estatuto da Criança e do Adolescente (ECA) (BRASIL, 2012).

Segundo o ECA (2012) considera-se criança, a pessoa até doze anos de idade incompletos, e adolescente aquela entre doze e dezoito anos de idade, além dos casos expressos em lei para pessoas entre dezoito e vinte e um anos de idade. O Estatuto da Criança e do Adolescente garante seguridade contra qualquer forma de negligência, discriminação, exploração, violência, crueldade e opressão, punindo na forma da lei qualquer atentado, por ação ou omissão, aos seus direitos fundamentais (saúde desde a gestação, educação). Desta forma, é obrigatória a notificação dos casos de maus tratos contra crianças e adolescentes, identificados por profissionais de saúde.

O ECA (2012) conta com o apoio do Conselho Tutelar para que seus direitos sejam cumpridos, oferecendo suporte e proteção para as vítimas e seus familiares, pois o campo de atuação deste órgão é amplo (saúde, educação, serviço social, previdência, trabalho e segurança). Assim, a parceria intersetorial entre o Conselho Tutelar e a Unidade Básica de Saúde tanto facilita, quanto fortalece a atuação dos profissionais envolvidos, que passa a ter suas responsabilidades compartilhadas. 
Segundo Hora TD e Paiva AR (2017) em 2010 a Secretaria de Atenção à Saúde criou o manual Linha de Cuidado para Atenção Integral à Saúde de Crianças, Adolescentes e suas Famílias em Situação de Violências - Orientação para Gestores e Profissionais de Saúde, para que o cuidado integral à saúde das vítimas e suas famílias fossem cumpridas, como também articular o cuidado desde à atenção básica até níveis mais complexos. Além disso, orienta a interação entre as demais esferas da sociedade para assegurar os direitos, proteção e defesa de crianças e adolescentes. Esse documento fornece questões relevantes para que os profissionais fortaleçam ações de prevenção à violência, promoção da saúde e cultura da paz, principalmente, junto às famílias. Além do mais, orienta os profissionais de saúde sobre a identificação precoce de sinais e sintomas de violência.

Apesar da proteção garantida por lei, no Brasil a violência é a principal causa de morte entre crianças e adolescentes a partir dos 5 anos de idade. De acordo com dados colhidos no período de 2006/2007 na base do Sistema de Vigilância de Violências e Acidentes (VIVA), a violência mais atendida nas unidades de saúde, contra crianças e adolescentes de 0 a 13 anos foi o estupro (BRASIL, 2010).

De acordo com Hora TD e Paiva AR (2017) em 2013 foram registradas 29.784 notificações de violências contra crianças na idade de 0 a 9 anos. Entre essas, 13.867 eram meninos, enquanto 15.917 eram meninas. Observou- se que a violência sexual foi a terceira causa mais notificada $(28,4 \%)$, sendo a negligência $(50,1 \%)$ a primeira causa mais registrada, seguida pela violência física $(28,6 \%)$. Em relação ao segmento com idade entre 10 a 19 anos registrou-se 50.634 notificações. Sendo 17.886 do sexo masculino e 32.748 do sexo feminino. A violência sexual aparece como a segunda causa de notificações $(23,9 \%)$, em primeiro lugar está a agressão física $(63,3 \%)$ e em terceiro, a violência psicológica/moral (23,0\%).

Hora TD e Paiva AR (2017) declaram que a violência sexual infanto juvenil no Brasil, relaciona-se ao patriarcalismo e machismo presentes na nossa sociedade, devido a desigualdade das relações entre mulheres e crianças e a sua submissão à figura masculina familiar. Diante disso, essa desestrutura social afeta as diversas esferas da sociedade, suas classes sociais e raças. Logo, a intervenção do Estado com a criação de políticas públicas voltadas para a garantia de proteção e cuidados para crianças e adolescentes tornou-se prioridade.

A Organização Mundial de Saúde (OMS) define violência sexual contra crianças e adolescente como qualquer envolvimento de caráter sexual com criança ou adolescente em que ele não compreende totalmente, seja incapaz de informar consentimento, não esteja preparada para desenvolvimento e consentimento do ato ou infrinja as leis ou tabus da sociedade (OMS,1999).

O abuso sexual de crianças e adolescente, abrange também os casos de assédio, estupro, pornografia infantil e exploração sexual, que podem se manifestar de diversas maneiras como: incesto, pedofilia, voyeurismo, penetração oral, anal ou genital com pênis ou objetos, jogos sexuais e práticas eróticas não consentidas. Inclui também, exposição coercitiva/constrangedora a atos libidinosos, exibicionismo, masturbação, linguagem erótica, interações sexuais de qualquer tipo (BRASIL, 2016).

Mais frequente que o câncer infantil, diabetes juvenil e doenças cardíacas congênitas, o abuso sexual infanto-juvenil ocorre em famílias de diversas raças, classes sociais e status econômico. Na maioria das vezes os abusos físicos se sobrepõem ao abuso sexual, longe de ser um incidente, geralmente, trata-se de abuso contínuo. O principal tipo de abuso é o intrafamiliar (causado por familiares próximos como pai, avô, irmão, primo, tio), mas pode ocorrer também em âmbito extrafamiliar (pessoas próximas da vítima) (RIBEIRO MA, et al., 2004).

Estudos demonstram que crianças e adolescente que são vítimas de abuso sexual possuem mais predisposição a desenvolver diversas patologias no decorrer de sua vida, como por exemplo sequelas psicológicas - como baixa autoestima, ansiedade, depressão, raiva, agressão, transtorno de estresse póstraumático (TEPT), dificuldades sexuais, baixo desempenho escolar, alcoolismo, abuso de drogas, depressão, tentativas de suicídio, dificuldades em manter gestações. Além disso, estão mais susceptíveis em adquirir Infecções Sexualmente Transmissíveis (ISTs) como a Síndrome da Imunodeficiência Adquirida (AIDS) (RIBEIRO MA, et al., 2004). 
Durante o período em que estão sendo vítimas dos abusos, os padecentes começam a apresentar sinais não habituais como agressividade, ansiedade na presença de algumas pessoas, raiva alterações de humor ou comportamento (RIBEIRO MA, et al., 2004). Além do mais, às vítimas de violência sexual durante a infância e adolescência podem apresentar também queixas somáticas, a exemplo de crises de falta de ar, irregularidade no ciclo menstrual mesmo que não tenha ocorrido penetração, problemas gastrointestinais sendo a dor abdominal aguda o mais frequente, distúrbios alimentares como bulimia e anorexia e alguns sintomas que podem acontecer associados ou de forma isolada como náuseas e vômitos, como também possuem mais predisposição a desenvolverem resistência a defecar (encoprese) e/ou vazamento involuntário de urina durante o sono ou em locais inadequados (enurese) (FLORENTINO BRB, 2015).

Kotzé JM e Brits H (2019) relatam que há três tipos de abuso sexual contra criança e adolescentes: (1) abuso sexual sem contato, onde a vítima pode sofrer ameaças de abuso, exposição a assédio sexual ou imagens sexuais visuais; (2) abuso sexual envolvendo relação sexual; e (3) entrar em contato com abuso sexual sem relação sexual, como tocar ou beijar inadequadamente. Florentino BRB (2015) relata que as sequelas do abuso sexual variam em cada indivíduo, e fatores como a idade em que ocorreu o abuso, a duração e a quantidade de vezes que ocorreu a violência, o grau da violência durante o abuso e se houve ameaças psicológica, podem impactar de forma significativa nas consequências trazidas pela agressão sexual.

Conforme Florentino BRB (2015) o abuso sexual infanto juvenil deixa marcas físicas, psíquicas, sociais e sexuais que comprometem a vida da vítima. Logo, a compreensão e a avaliação das sequelas do abuso tornase difícil. Os efeitos da violência sexual podem aparecer em qualquer idade da vida, sendo um gatilho para o desenvolvimento de psicopatologias que interferem no desenvolvimento psicológico, afetivo e emocional. A avaliação da vítima de abuso sexual deve levar em consideração as particularidades que envolveram a violência, como se houve penetração e o grau dessa penetração, se ocorreu violência psicológica e / ou física.

De acordo com Azambuja MRF e Ferreira MH (2011), apesar da notificação ser compulsória, por força da lei, ainda é muito incipiente o registro/disponibilização de dados para mensurar o tamanho real do fenômeno da violência sexual contra menores, em razão da subnotificação. Segundo especialistas, apenas 10\% dos casos são notificados às autoridades. Um problema enfrentado pelos profissionais de saúde no combate ao abuso sexual de crianças e adolescentes é a busca tardia por atendimento médico. Outro problema é que a maioria das crianças vítimas de abuso sexual não apresenta achados físicos anormais, mesmo nos casos penetrantes devido a sua fisiologia de crescimento e desenvolvimento estar em constante progressão, suas feridas recuperam sem deixar cicatrizes, o que dificulta ainda mais a comprovação do crime.

$O$ atendimento das vítimas infanto juvenil de violência sexual no âmbito da saúde, deve se basear no acolhimento multiprofissional da vítima, associado ao encaminhamento para a rede de cuidados necessários, em que são realizadas consulta clínica com anamnese, exame físico, exames complementares, além de realizar o planejamento para tratamento e profilaxia de doenças, como também a avaliação psicológica (HORA TD e PAIVA AR, 2017).

Uma anamnese bem direcionada orienta o exame físico, facilita a coleta de amostras médico-legal, além de proteger as vítimas de abusos recorrentes. Embora o exame físico seja importante, o diagnóstico de abuso sexual infantil, geralmente, é baseado nas declarações da vítima, as quais devem ser obtidas através de procedimento adequado. Para que as crianças vítimas de abuso sexual possam receber assistência médica apropriado e oportuno, os médicos envolvidos devem ter o conhecimento necessário nas áreas de ginecologia infantil/adolescente e medicina forense, além de contar com uma equipe multiprofissional capaz de oferecer suporte adequado para às vítimas e seus familiares (KOTZÉ JM e BRITS H, 2019).

O exame físico deve ser realizado de forma completa com atenção especial para boca, mamas, genitais, região perineal, nádegas e ânus. Os principais achados nas vítimas de abuso incluem, hiperemia, escoriações, edema, hematomas e até danos com maior grau de severidade como, lesões genitais causadas não somente pela penetração, mas também pela introdução de objetos e dos dedos do abusador no interior da vagina da vítima, sangramento de hímen, irritação e lesão da mucosa vaginal, corrimento vaginal, cicatriz genital e lesões anais como laceração, sangramento, perda do controle esfincteriano. O profissional de saúde 
deve atentar-se para o fato de que fatores diversos como idade, níveis hormonais, variações morfológicas normais, impossibilitam testar o hímen das meninas. Já para meninos, lesões penianas, escrotais ou testiculares são causadas, acidentalmente, visto que a maioria dos agressores evitam causar ferimentos para não serem descobertos (CAO Z, et al., 2019).

$\mathrm{Na}$ existência de lesão anal, deve-se interpretar de formar cuidadosa o seu relaxamento e dilatação, pois achados semelhantes são encontrados em outras situações como doenças neurológicos, constipação crônica, sedação e paralisia. Por outro lado, os achados negativos do ânus e órgãos genitais não podem excluir a possibilidade de abuso sexual invasivo (PELISOLI C, et al., 2010). Além das áreas da região íntima, deve-se avaliar a vítima por completo, pois pode haver lesões físicas associadas, como ferimentos por tentativa de enforcamento, contusões e fraturas, queimaduras causadas por cigarro que manifestam 0 sadismo do agressor (FLORENTINO BRB, 2015).

Segundo informações da Sociedade Brasileira de Pediatria (SBP) (2001) além da anamnese e do exame físico, sempre que possível devem ser solicitados exames complementares para auxiliar na comprovação do abuso, na identificação de ISTs ou gestação em vítimas púberes. Alguns exames quando realizados até 72 horas após o ocorrido, podem fornecer material biológico do agressor em pubescentes. Em vítimas que ainda não entraram na puberdade, a maioria dos achados biológicos são encontrados nas suas vestes ou roupas de cama.

Além do atendimento médico minucioso, o profissional deve notificar imediatamente os casos para a Secretaria Municipal de Saúde através da ficha de notificação do Sistema de Vigilância de Violências e Acidentes (VIVA), como também comunicar o Conselho Tutelar para que seja feito o acompanhamento do caso de forma contínua. Em casos em que seja necessário realizar procedimentos que são proibidos na legislação vigente no país, como a interrupção de gestação, deve-se acionar o Ministério Público (HORA TD e PAIVA AR, 2017).

Ademais, as vítimas devem receber apoio psicológico contínuo, visto que a dificuldade em verbalizar os fatos é explícita. Nos casos em que os agressores são do meio familiar, o silêncio ainda é mais frequente, pois elas temem sofrer castigos por ambos os genitores ou sentem-se responsáveis em manter o equilíbrio e integridade da família. Diante dessa situação, às vítimas sentem-se desamparadas e culpadas pelo ocorrido (FLORENTINO BRB, 2015).

Diante disso, a criança ou adolescente vítima de abuso sexual que tem a oportunidade de verbalizar o ocorrido recebendo apoio profissional e familiar, conjuntamente, tem melhor prognóstico no desaparecimento das manifestações mais evidentes, podendo a vítima reencontrar interesse por si, pelo convívio com os outros, interagir em brincadeiras que antes gostava. Entretanto, pode ocorrer que as angústias pelo o ocorrido se manifestem de outras formas como, fobias (medo do escuro, agorafobia, afastamento do ciclo familiar, dos amigos, de pessoas do mesmo sexo, solidão), paranoia, sensação de vazio e não pertencimento, insônia (FLORENTINO BRB, 2015).

\section{CONSIDERAÇÕES FINAIS}

A revisão literária utilizada neste trabalho permitiu observar que apesar da existência de políticas públicas voltadas para o combate à violência sexual contra crianças e adolescentes, existem fatores que dificultam o diagnóstico precoce, assim como uma possível intervenção. Dentre estes fatores, podem ser destacadas a escassez de profissionais capacitados no assunto e a subnotificação dos casos. Neste contexto, o médico generalista e, em especial, o médico da estratégia de saúde da família, devido a sua proximidade de contato e ao vínculo estabelecido com a população adscrita do território sob sua responsabilidade, constitui-se numa peça fundamental de atuação no combate ao crime sexual contra menores. Sua atenção longitudinal junto às famílias permitiria a identificação precoce de eventos suspeitos, associada ao apoio e capacitação dos profissionais que compõem a sua equipe, a respeito este complexo tema. Para tal, o médico também precisa se habilitar/qualificar acerca da fisiopatologia e dos aspectos legais que envolvem a saúde integral da criança e do adolescente. 


\section{REFERÊNCIAS}

1. AZAMBUJA MRF, MARIANTE MH. Violência sexual contra crianças e adolescentes. Porto Alegre: Artmed, 2010; 392p.

2. BRASIL. Constituição Federal de 1988. Constituição da República Federativa do Brasil. Presidência da República: Brasília - DF, 1988. Disponível em: http://www.planalto.gov.br/ccivil_03/constituicao/constituicao.htm. Acesso em: 20 abr. 2020.

3. BRASIL. Lei o 8069 de 13 de julho de 1990. Dispõe sobre o Estatuto da Criança e do Adolescente e dá outras providências. Diário Oficial da União: Brasília - DF, 1990. Disponível em: http://www.planalto.gov.br/ccivil_03/leis//8069.htm. Acesso em: 20 abr. 2020.

4. BRASIL. Lei o 9.970 de 17 de maio de 2000. Institui o dia 18 de maio como o Dia Nacional de Combate ao Abuso e à Exploração Sexual de Crianças e Adolescentes. Diário Oficial da União: Brasília - DF, 2000. Disponível em: http://www.planalto.gov.br/ccivil_03/leis//9970.htm. Acesso em: 20 abr. 2020.

5. BRASIL. Ministério da Saúde. Análise epidemiológica da violência sexual contra crianças e adolescentes no Brasil, 2011 a 2017. Boletim epidemiológico, Brasília, 2018; 49(27).

6. BRASIL. Ministério da Saúde. Impacto da violência na saúde de crianças e adolescentes. Brasília, 2010. Disponível em: http://bvsms.saude.gov.br/bvs/publicacoes/impacto_violencia_saude_criancas_adolescentes.pdf. Acesso em: 20 abr. 2020.

7. BRASIL. Ministério da Saúde. Portaria no 2.436, de 21 de setembro de 2017. Aprova a Política Nacional de Atenção Básica, estabelecendo a revisão de diretrizes para a organização da Atenção Básica, no âmbito do Sistema Único de Saúde (SUS). Diário Oficial da União: Brasília - DF, 2017. Disponível em: https://bvsms.saude.gov.br/bvs/saudelegis/gm/2017/prt2436_22_09_2017.html. Acesso em: 22 ago. 2020.

8. BRASIL. Ministério da Saúde. VIVA: Instrutivo - Notificação de Violência Interpessoal e Autoprovocada. $2^{\mathrm{a}}$ edição. Brasília: Ministério da Saúde, 2016. Disponível em: http://bvsms.saude.gov.br/bvs/publicacoes/viva_instrutivo_violencia_interpessoal_autoprovocada_2ed.pdf. Acesso em: 20 ago. 2020.

9. CAO Z, et al. Forensic identification of child sexual abuse. Journal of Forensic Medicine, 2019. 35(6): 733-736.

10. COSTA F. Longe da escola, crianças vítimas de violência podem não ter para quem pedir ajuda durante o isolamento. Universidade Federal do Rio Grande do Sul (UFRGS), Jornal da Universidade, 2020. Disponível em: https://www.ufrgs.br/jornal/longe-da-escola-criancas-vitimas-de-violencia-podem-nao-ter-para-quemlonge-daescola-criancas-vitimas-de-violencia-podem-nao-ter-para-quem-pedir-ajuda-no-periodo-de-isolamento-social/. Acesso em: 20 maio 2020.

11. COSTA MCO, et al. O perfil da violência contra crianças e adolescentes, segundo registro de conselheiros tutelares: vítimas, agressores e manifestações de violência. Ciểncia e saúde coletiva, 2007; 12 (5): 1129-1141.

12. FLORENTINO BRB. As possíveis consequências do abuso sexual praticado contra crianças e adolescentes. Fractal: Revista de Psicologia, 2015; 27(2): 139-144.

13. HABIGZANG LF, et al. Fatores de risco e de proteção na rede de atendimento a crianças de adolescentes vítimas de violência sexual. Psicologia: reflexão e crítica, 2006; 19(3): 379-386.

14. HORA TD, PAIVA AR. Violência sexual contra crianças e adolescentes no campo da saúde e a intersetorialidade no sistema de garantias de direitos. Sustinere, 2017; 5(2): 296-316.

15. KOTZÉ JM, BRITS H. Child sexual abuse: the significance of the history and testifying on non-confirmatory findings. Afr J Prm Health Care Fam Med, 2019; 11 (1): 2071-2936.

16. LIMA JS, DESLANDES SF. A notificação compulsória do abuso sexual contra crianças e adolescentes: uma comparação entre os dispositivos americanos e brasileiros. Interface, 2011; 15(38): 819-832

17. MINAYO MCS. Violência contra criança e adolescentes: questão social, questão de saúde. Revista Brasileira Saúde Materno Infantil, 2001; 1(2): 91- 102.

18. ORGANIZAÇÃO MUNDIAL DA SAÚDE (OMS). Relatório da Consulta sobre Prevenção ao Abuso Infantil da Organização Mundial da Saúde, 1999. Disponível em: https://apps.who.int/iris/bitstream/handle/10665/207717/9789241565356-por.pdf?ua=1. Acesso em 14 ago. 2020.

19. PELISOLI C, et al. Violência sexual contra crianças e adolescentes: dados de um serviço de referência. Temas em Psicologia, 2010; 18(1): 85-97.

20. RIBEIRO MA, et al. Violência sexual contra crianças e adolescentes: características relativas à vitimização nas relações familiares. Caderno de Saúde Pública,2004; 20(2): 456 - 464.

21. SANTOS CO, et al. Violência contra crianças e adolescentes: análise sócio - histórica do desenvolvimento da violência no processo de sociabilidade do homem. Desafios Contemporâneos,2015; 8(1): 286 -292.

22. SOCIEDADE BRASILEIRA DE PEDIATRIA (SBP). Guia de atuação frente a maus-tratos na infância e na adolescência. $2^{\mathrm{a}}$ Edição. Rio de Janeiro: 2001.2 Disponível em: https://www.sbp.com.br/fileadmin/user_upload/maustratos_sbp.pdf Acesso em: 20 abr. 2020 